\title{
Novel biomarker candidates for the diagnosis of ovarian clear cell carcinoma (Review)
}

\author{
HIROSHI KOBAYASHI, HITOMI SUGIMOTO, SHUNSUKE ONISHI and KAZUTOSHI NAKANO \\ Department of Obstetrics and Gynecology, Nara Medical University, Kashihara, Nara 634-8522, Japan
}

Received September 28, 2014; Accepted May 27, 2015

DOI: $10.3892 / \mathrm{ol} .2015 .3367$

\begin{abstract}
Ovarian clear cell carcinoma can arise from endometriosis; however, it is distinct from other types of epithelial ovarian carcinoma in terms of its clinicopathological and molecular features. Cancer antigen 125 lacks the sensitivity and specificity required for accurate clinical diagnosis of clear cell carcinoma. Therefore, the aim of the current review was to identify novel biomarker candidates for the immunohistochemical and serological diagnosis of clear cell carcinoma. A search of the relevant English language literature published between 1966 and 2014 was conducted using the PubMed MEDLINE online database. High-throughput tissue microarray technology and proteomic screening combined with mass spectrometry may provide additional information regarding diagnostic biomarker candidates for ovarian clear cell carcinoma. The present review summarizes the characteristics of potential genomic alterations that activate cancer signaling pathways and, thus, contribute to carcinogenesis. The major signaling pathways activated in clear cell carcinoma are associated with cell cycle regulation (hepatitis A virus cellular receptor 1 and tumor protein D52), growth factor signaling (insulin-like growth factor binding protein 1; KiSS-1 metastasis-suppressor; erb-b2 receptor tyrosine kinase 2; and fibroblast growth factor receptor 2), anti-apoptosis and survival pathways [sialidase 3 (membrane sialidase)], metabolism ( $\gamma$-glutamyltransferase 1$)$, chemoresistance (napsin A aspartic peptidase, glutathione peroxidase 3; and aldehyde dehydrogenase 1 family, member A1), coagulation [coagulation factor III (thromboplastin, tissue factor); and tissue factor pathway inhibitor 2], signaling (lectin, galactoside-binding and soluble, 3), and adhesion and the extracellular matrix [cadherin 1, type 1, E-cadherin (epithelial); versican; and laminin, $\alpha$ 5]. The present review of the relevant literature may provide a basis for additional clinical investigation of the ovarian clear cell carcinoma serum biomarker candidate proteins identified herein.
\end{abstract}

Correspondence to: Professor Hiroshi Kobayashi, Department of Obstetrics and Gynecology, Nara Medical University, 840 Shijo-Cho, Kashihara, Nara 634-8522, Japan

E-mail: hirokoba@naramed-u.ac.jp

Key words: ovarian clear cell carcinoma, biomarker, gene expression profiling, proteomics

\section{Contents}

1. Introduction

2. Search strategy and selection criteria

3. Overexpression of genes and their transcripts in ovarian clear cell carcinoma

4. Additional biomarker candidates in ovarian clear cell carcinoma

5. Discussion

\section{Introduction}

Epithelial ovarian carcinoma is commonly diagnosed at an advanced stage due to a lack of effective serum markers. For example, the conventional serum cancer antigen 125 (CA125) is a poor marker for early diagnosis (1). CA125 is elevated in the serum of $>80 \%$ of patients with high-grade serous adenocarcinoma, but false-negative results for CA125 frequently occur in cases of ovarian clear cell carcinoma (2). However, CA125 is the only currently used diagnostic marker for ovarian clear cell carcinoma. Ovarian clear cell carcinoma occurs more frequently in Japan than in Western countries (3). It is considered to arise from endometriosis (4). Compared with other histological types, clear cell carcinoma has been recognized to display a chemoresistant phenotype, which leads to poorer prognosis (3); its response rate to paclitaxel plus carboplatin therapy is lower compared with other histological types, ranging from $22-56 \%$. Therefore, the identification and development of more accurate clear cell carcinoma diagnostic markers is required.

Epithelial ovarian carcinoma is a heterogeneous disease with various histological subtypes, including serous, mucinous, endometrioid and clear cell. Each histological subtype is a classified as a separate disease with characteristic cytogenetic features, molecular signatures, oncogenic signaling pathways and clinicobiological behaviors (5). Epithelial ovarian carcinomas are classified into two major subtypes, types I and II, based on their distinctive epigenetic and gene expression profiles, in addition to their functional genomic mutations. Type I carcinomas include low-grade serous, mucinous, endometrioid and clear cell subtypes. They evolve from precursor lesions (typically borderline tumors) in a slow, step-wise process, with prolonged early-stage disease and a more favorable prognosis than type II high-grade tumors (5). 
Type I carcinomas frequently exhibit mutations in the following genes: AT-rich interactive domain 1A (SWI-like) (ARID1A); phosphatidylinositol-4,5-bisphosphate 3-kinase, catalytic subunit $\alpha$ (PIK3CA); protein phosphatase 2 regulatory subunit A- $\alpha$; B-Raf proto-oncogene, serine/threonine kinase; Kirsten rat sarcoma viral oncogene homolog; phosphatase and tensin homolog; and catenin (cadherin-associated protein), $\beta 1,88 \mathrm{kDa}$. In addition, type I epithelial ovarian carcinomas lack mutations in tumor protein p53 (TP53). By contrast, type II carcinomas originate de novo from the adnexal epithelia with no identifiable precursor lesion, develop rapidly and are of an aggressive nature. Type II tumors include high-grade serous, endometrioid, mixed and undifferentiated carcinomas (5). Mutations in TP53 are common in type II carcinomas and this distinction may serve as a marker of malignant growth (6).

There is a requirement for the development of novel serum markers for clear cell carcinoma to improve its early diagnosis, therapeutic stratification and prognosis, as well as for monitoring patients that have undergone treatment. The proteomic and genome-wide search techniques available for exploring sensitive and specific biomarkers may facilitate the early diagnosis of clear cell carcinoma in the future. The current review presents novel biomarker candidates obtained from a diverse cohort of patients diagnosed with ovarian clear cell carcinoma, and proposes that specific, overexpressed tumor self-antigens may be used as diagnostic serum marker candidates.

\section{Search strategy and selection criteria}

The current study aimed to identify markers for distinguishing clear cell carcinoma from high-grade serous adenocarcinoma. A literature review was conducted to investigate the molecular phenotype of ovarian clear cell carcinoma-specific genes. A PubMed MEDLINE (http://www.ncbi.nlm.nih. gov/pubmed) search of the relevant literature published between 2005 and 2014 was performed using the following key words: 'Epithelial ovarian cancer', 'clear cell carcinoma', 'gene expression profiling', 'proteome' and 'marker'. English-language publication search results from PubMed and references within the relevant articles were analyzed. Furthermore, references within the references were searched to identify additional relevant studies.

A list of genes and their transcripts significantly differentially expressed in patients with clear cell carcinoma versus high-grade serous adenocarcinoma was created. Genes and their encoded proteins identified in two or more of the primary studies are listed on Table I. To minimize selection bias, screening of the studies was independently performed by two of the co-authors (HS and SO) after agreeing on the selection criteria.

A total of 2087 articles were identified by the search; around 350 articles were potentially relevant. Only 91 publications available for distinguishing clear cell carcinoma from high-grade serous adenocarcinoma were chosen based on the final selection, taking into account the title and the summary analysis. Others were excluded due to various reasons, including selection bias, detection bias, reporting bias and other possible sources of bias.

\section{Overexpression of genes and their transcripts in ovarian clear cell carcinoma}

Gene expression profiling and proteomic analysis have been used in previous studies to evaluate levels of gene and protein expression, respectively, in the following two subtypes of epithelial ovarian carcinoma: High-grade serous carcinoma and clear cell carcinoma. These have been characterized by gene and protein expression patterns of specific tissue markers, allowing objective detection of each disease. Furthermore, it was identified that atypical endometriosis and clear cell carcinoma share molecular alterations, such as inactivating mutations for ARID1A, activating mutations for PIK3CA and the hypomethylation of HNF1 homeobox B (HNF1B) (4). The HNF1B protein binds to DNA as a member of the homeodomain-containing superfamily of transcription factors. This transcription factor plays a role in endometrial regeneration, differentiation, decidualization, glycogen synthesis, detoxification, cell cycle regulation, implantation, uterine receptivity and a successful pregnancy (7). Furthermore, HNF1B regulates a subset of progesterone target genes and may act as a modulator of female reproduction (7). The immunohistochemical study revealed that the positive $\mathrm{HNF} 1 \mathrm{~B}$ staining was a frequent finding in clear cell carcinoma. Ovarian clear cell carcinomas exhibit increased activity of signaling pathways involved in cell cycle regulation, survival, anti-apoptosis, chemoresistance, metabolism, coagulation and angiogenesis (7). Table I includes possible clear cell carcinoma-specific biomarkers identified using the aforementioned search strategy (Table I).

Cell cycle regulation. Clear cell carcinoma exhibits a unique pattern of expression of cell cycle regulatory molecules among epithelial ovarian carcinomas (8). HNF1B induces cell cycle arrest and stimulates an anti-apoptotic response to oxidative stress-induced DNA damage (9). Therefore, elucidating the detailed association between HNF1B upregulation and the acquisition of cell cycle regulation under conditions of oxidative stress may improve understanding of the mechanisms used to repair DNA damage (7). A previous study identified the role of the HNF1B-mediated signaling pathway in a fail-safe mechanism for the oxidative stress-induced DNA damage response (10). The present study identified six genes, F-Box protein $5(11,12)$, Cdk5 and Abl enzyme substrate 1 (13), hepatitis A virus cellular receptor 1 (HAVCR1) (14), cyclin E1 (15), tyrosine 3-monooxygenase/tryptophan 5-monooxygenase activation protein, $\theta(16)$ and TPD52 $(17,18)$, associated with regulation of the cell cycle regulation that are abnormally expressed in ovarian clear cell carcinoma. Among them, HAVCR1 $(14)$ and TPD52 $(17,18)$ have been proposed as serum marker candidates in ovarian clear cell carcinoma.

Cell proliferation and survival. The growth factor receptor axis is frequently dysregulated in a wide variety of human cancer types via gene amplification, mutation, protein overexpression and aberrant activation. The overexpression of growth factor receptors has been associated with potentially aggressive tumors and correlated with poor prognosis. Microarray analysis and proteome studies identified a number of candidate marker genes for cell proliferation and survival in ovarian clear cell carcinoma, including PIK3CA $(6,19,20)$, 
Table I. Genes recognized as ovarian clear cell carcinoma-specific biomarkers.

Symbol

Full gene name

Proposed as a serum

marker candidate

References

Cell cycle regulation

\section{FBXO5 \\ CABLES1 \\ HAVCR 1 \\ CCNE1 \\ YWHAQ}

TPD5

Cell proliferation and survival

\section{PIK3CA}

MET
IGFBP1
IGF2BP3
PDGFRA
KISS1
KISS1R
ERBB2
FGFR2
ARHGDIA
PAX8

Anti-apoptosis

HNF1B
PPM1D
APPBP2

BCL2

NEU3

Metabolism

HNF1B

GGT1

VHL

MUC1

MAP1LC3A

HOXA10

Chemoresistance

XIAP

NAPSA

PPP1R13L

GPC3

$\mathrm{ABCF} 2$

GPX3

ANXA4

ALDH1A1

Coagulation

TFPI2

F3

Angiogenesis

VEGFA
F-box protein 5

10-12

Cdk5 and Abl enzyme substrate 1

Hepatitis A virus cellular receptor 1

Cyclin E1

tyrosine 3-monooxygenase/tryptophan

5 -monooxygenase activation protein, $\theta$

Tumor protein D52

Yes

8,13

Yes

14

15

16

17,18

Phosphatidylinositol-4,5-bisphosphate 3-kinase,

$4,19,20$

catalytic subunit $\alpha$

MET proto-oncogene, receptor tyrosine kinase

21

Insulin-like growth factor binding protein 1

22,23

Insulin-like growth factor 2 mRNA binding protein 3

24

Platelet-derived growth factor receptor, $\alpha$ polypeptide

25,26

KiSS-1 metastasis-suppressor

KISS1 receptor

27,28

27,28

Erb-b2 receptor tyrosine kinase 2

Fibroblast growth factor receptor 2

29

Rho GDP dissociation inhibitor (GDI) $\alpha$

Paired box 8

30,31

32,33

34

HNF 1 homeobox B

Protein phosphatase, $\mathrm{Mg}^{2+} / \mathrm{Mn}^{2+}$ dependent, 1D

$4,35,36$

37

38

binding protein 2

B-cell CLL/lymphoma 2

39

Sialidase 3 (membrane sialidase)

Yes

40,41

HNF 1 homeobox B

43,44

$\gamma$-glutamyltransferase 1

von Hippel-Lindau tumor suppressor, E3 ubiquitin

protein ligase

Mucin 1, cell surface associated

Microtubule-associated protein 1 light chain $3 \alpha$

Homeobox A10

Yes

45

40,46

47

42

48

X-linked inhibitor of apoptosis, E3 ubiquitin protein

ligase

Napsin A aspartic peptidase

Protein phosphatase 1, regulatory subunit 13 like

Glypican 3

ATP-binding cassette, sub-family F (GCN20), member 2

Glutathione peroxidase 3

Annexin A4

Aldehyde dehydrogenase 1 family, member A1

Yes

50

51

52,53

54

Yes

55

56

Yes

57

Tissue factor pathway inhibitor 2

Coagulation factor III (thromboplastin, tissue factor)

Yes

59,60

Yes

Vascular endothelial growth factor A 
Table I. Continued.

\begin{tabular}{|c|c|c|c|}
\hline Symbol & Full gene name & $\begin{array}{l}\text { Proposed as a serum } \\
\text { marker candidate }\end{array}$ & References \\
\hline \multicolumn{4}{|c|}{ Angiogenesis } \\
\hline HYAL1 & Hyaluronoglucosaminidase 1 & & 62 \\
\hline CXCR4 & Chemokine (C-X-C motif) receptor 4 & & 63 \\
\hline CXCL12 & Chemokine (C-X-C motif) ligand 12 & & 63 \\
\hline THBS2 & Thrombospondin 2 & & 64 \\
\hline \multicolumn{4}{|c|}{ Chromatin remodeling } \\
\hline ARID1A & AT rich interactive domain 1A (SWI-like) & & 4,66 \\
\hline \multicolumn{4}{|l|}{ Signaling } \\
\hline HILPDA & Hypoxia inducible lipid droplet-associated & & 67 \\
\hline LGALS3 & Lectin, galactoside-binding, soluble, 3 & Yes & 12,68 \\
\hline SFRP5 & Secreted frizzled-related protein 5 & & 69 \\
\hline \multicolumn{4}{|c|}{ Adhesion and extracellular matrix } \\
\hline MSLN & Mesothelin & & 70 \\
\hline $\mathrm{CDH} 1$ & Cadherin 1, type 1, E-cadherin (epithelial) & Yes & 71 \\
\hline VCAN & Versican & Yes & 72,73 \\
\hline LAMA5 & Laminin, $\alpha 5$ & Yes & 74 \\
\hline \multicolumn{4}{|l|}{ Hormone } \\
\hline ER & Estrogen receptor & & 4,75 \\
\hline PR & Progesterone receptor & & 4,75 \\
\hline \multicolumn{4}{|l|}{ Mucin } \\
\hline PDPN & Podoplanin & & 76 \\
\hline
\end{tabular}

MET proto-oncogene, receptor tyrosine kinase (21), insulin-like growth factor binding protein 1 (IGFBP1) $(22,23)$, IGF2 mRNA BP3 (24), platelet-derived growth factor receptor, $\alpha$ polypeptide $(25,26), \mathrm{KiSS}-1$ metastasis-suppressor (KISS1) (27,28), KISS1 receptor $(27,28)$, Erb-b2 receptor tyrosine kinase 2 (ERBB2) (29), Erb-b2 receptor tyrosine kinase 2 (FGFR2) (30,31), Rho GDP dissociation inhibitor (GDI) $\alpha(32,33)$ and paired box 8 (34). In particular, additional clinical studies are required to assess the efficacy of circulating IGFBP1, KISS1, ERBB2 and FGFR2 protein expression for the diagnosis of ovarian clear cell carcinoma.

Anti-apoptosis. Genes involved in anti-apoptosis have previously been demonstrated to undergo epigenomic and genomic changes in clear cell carcinoma. HNF1B is involved in carcinogenesis through the upregulation of genes involved in anti-apoptosis. The present study identified key genes involved in anti-apoptosis in ovarian clear cell carcinoma, including $\mathrm{HNF} 1 \mathrm{~B}(6,35,36)$, protein phosphatase, $\mathrm{Mg}^{2+} / \mathrm{Mn}^{2+}$ dependent, 1D (37), amyloid $\beta$ precursor protein (cytoplasmic tail) binding protein 2 (38), B-cell CLL/lymphoma 2 (39) and sialidase 3 (membrane sialidase) (NEU3) $(40,41)$. As NEU3 is a cell surface protein, it may be a good serum marker candidate for the detection of ovarian clear cell carcinoma $(40,41)$.

Metabolism. Glucose metabolism is reprogrammed to generate energy by glycolysis in cancer cells and is one of the key clinical features of carcinoma used to facilitate cancer cell survival. The ovarian clear cell carcinoma histotype exhibits specific metabolic features in which tumor growth is dependent on anaerobic glycolysis as opposed to oxidative phosphorylation for rapid energy generation, a process termed the Warburg effect. This metabolic characteristic also confers resistance to apoptosis (42). Numerous genes are involved in metabolism in ovarian clear cell carcinoma, including HNF1B $(43,44)$, $\gamma$-glutamyltransferase 1 (GGT1) (45), von Hippel-Lindau tumor suppressor, E3 ubiquitin protein ligase $(44,46)$, mucin 1, cell surface associated (47), microtubule-associated protein 1 light chain $3 \alpha$ (42) and homeobox A10 (48). In particular, GGT1 may be a candidate serum marker for diagnosing ovarian clear cell carcinoma.

Chemoresistance. Biologically diverse mechanisms are involved in the development of a chemoresistant phenotype in ovarian clear cell carcinoma. These include the activation of anti-apoptosis, detoxification and antioxidation systems in the cell. The resistance of ovarian clear cell carcinoma to platinum-based chemotherapy may be caused by low levels of cell proliferation and increased cell cycle arrest during the response to DNA damage. A number of up- and downregulated chemoresistance genes have been categorized into differentially-expressed functional groups. The expression of eight genes, X-linked inhibitor of apoptosis, E3 ubiquitin protein ligase (49), Napsin A aspartic peptidase (NAPSA) (50), protein phosphatase 2 regulatory subunit 13 like $(51)$, glypican $3(52,53)$, ATP-binding cassette, sub-family F (GCN20), member 2 (54), glutathione peroxidase 3 (55), annexin A4 (56) and aldehyde dehydrogenase 1 family, member A1 (ALDH1A1) (57), were 
demonstrated to be upregulated in ovarian clear cell carcinoma specimens. Among these eight genes, NAPSA, GPX3 and ALDH1A1 were markedly overexpressed and may be possible novel biomarker candidates.

Coagulation. HNF1B is a broad marker of the ovarian clear cell carcinoma phenotype (58). Microarray analysis conducted in a previous study revealed that HNF1B may be associated with the blood clotting signaling cascade, which includes the overexpression of fibrinogen, prothrombin, coagulation factor III (thromboplastin, tissue factor) (F3) and factor XIII. Thus, HNF1B acts as a molecular link between the ovarian clear cell carcinoma histotype and an increase in the risk of developing clinically significant venous thromboembolism (VTE) (43). In particular, the coagulation-associated biomarkers F3 and tissue factor pathway inhibitor 2 (TFPI2) may be useful for the detection of clear cell carcinoma $(59,60)$. For example, tumor-derived F3 may initiate an extrinsic coagulation signaling cascade, triggering VTE formation in clear cell carcinoma.

Angiogenesis. Angiogenesis-associated signaling pathways are potentially the biological activity most associated with an ovarian clear cell carcinoma signature. Furthermore, analysis of gene expression data in previous studies demonstrated that vascular endothelial growth factor A (61), hyaluronoglucosaminidase 1 (62), chemokine (C-X-C motif) (CXC) receptor 4 (63), CXC ligand 12 (63) and thrombospondin 2(64) constitute the angiogenesis-associated signaling most significantly associated with upregulated gene expression in ovarian clear cell carcinoma.

\section{Additional biomarker candidates in ovarian clear cell carcinoma}

Chromatin remodeling. Chromatin remodeling may be important for the repair of DNA damage in oxidative lesions, in order to maintain genome stability (65). Components of the chromatin remodeling multiprotein complexes may harbor develop gene sequence mutations that lead to protein inactivation or loss of expression in various types of human cancer, including ovarian clear cell carcinoma (66).

Cancer-associated signaling pathways. Previous studies have identified a number of up- and downregulated genes involved in signaling pathways associated with clear cell carcinoma (12,67-69). One of these genes, lectin, galactoside-binding, soluble, 3 (LGALS3), is a potential serum biomarker for the diagnosis of ovarian clear cell carcinoma.

Adhesion and the extracellular matrix. Previous studies have revealed the clinical importance of certain cellular adhesion molecules for the diagnosis and prognosis of patients with ovarian clear cell carcinoma. These include mesothelin (70), cadherin 1, type 1, E-cadherin (epithelial) (CDH1) (71), versican (VCAN) (72,73) and laminin, $\alpha 5$ (LAMA5) (74), the last three of which are serum biomarker candidates.

Hormones. Ovarian clear cell carcinoma is a subtype of epithelial ovarian carcinoma characterized by an estrogen- and progesterone receptor-negative phenotype (75). Thus, this phenotype may be used as an immunohistochemical diagnostic marker for patients with ovarian clear cell carcinoma.

Mucin. In addition, mucin is key in the process of malignant transformation via promotion of cell invasion, metastasis and increased aggressiveness of cancer cells (76). Among the mucin-type characteristics, podoplanin expression was significantly stronger in clear cell carcinoma than in other histological types (76). Although elevated mucin expression was considered to be a prognostic marker for ovarian cancer, no significant correlation was observed between podoplanin expression and overall survival. Further investigation is needed to clarify the relationship between podoplanin expression and the biological characteristics of clear cell carcinoma.

\section{Discussion}

To date, the early detection of ovarian clear cell carcinoma has been hindered by the absence of effective serum biomarkers. Previous studies have failed to identify a biomarker panel with significantly improved performance over CA125. Technological advances have permitted the development of novel methods for identifying genes and proteins responsible for disease predisposition. In particular, high-throughput techniques using microarray technology and proteomic screening have generated a collection of marker candidates for use in the diagnosis of ovarian clear cell carcinoma. Ovarian clear cell carcinoma exhibits increased activity of the following signaling pathways known to potentially drive carcinogenesis: Cell cycle regulation, survival, anti-apoptosis, chemoresistance, metabolism, coagulation and angiogenesis (7). The current review summarizes characteristics of each genomic alteration that results in activation of the aforementioned cancer signaling pathways, as well as other potential sources of diagnostic and prognostic biomarkers for ovarian clear cell carcinoma.

The present review highlights specific genes and their products that are aberrantly expressed in ovarian clear cell carcinoma tissues, thus indicating their role as potential serum markers for the diagnosis of the disease. Biomarkers identified in the current review are involved in cell cycle regulation (HAVCR1 and TPD52), growth factor signaling (IGFBP1, KISS1, ERBB2 and FGFR2), anti-apoptosis and survival pathways (NEU3), metabolism (GGT1), chemoresistance (NAPSA, GPX3 and ALDH1A1), coagulation (F3 and TFPI2), signaling (LGALS3), and adhesion and the extracellular matrix (CDH1, VCAN and LAMA5; Table I).

HAVCR1 functions as part of the regulatory apparatus for tight junctions and may serve as a marker for renal and ovarian clear cell carcinoma susceptibility, as well as tumor invasion and metastasis (14). Furthermore, the physiological function of TPD52 may be associated with cell proliferation and the generation of a metastatic phenotype (17). IGFBP1, KISS1, ERBB2 and FGFR2 regulate the functions of growth factors that may be molecular targets in patients with ovarian clear cell carcinoma, while NEU3 protects cancer cells from apoptosis and is a marker of favorable prognosis (40). In addition, GGT1 has been implicated in glucose and lipid metabolism (45). NAPSA and ALDH1A1 are correlated with resistance to certain anti-cancer agents (50); GPX3 acts as a potent tumor suppressor by decreasing the number of 
reactive oxygen species and limiting DNA damage (55); F3 and TFPI 2 have roles in the regulation of the extrinsic coagulation cascade and thrombosis $(59,60)$; and LGALS3 is associated with the $\mathrm{Wnt} / \beta$-catenin signaling pathway, which is important oncogenesis $(12,68)$. Furthermore, CDH1 predicts a favorable prognosis (71). Thus, the current review may provide a basis for further clinical investigation of the aforementioned serum marker candidates. So far, there has been no data concerning the relationship between the expression of the identified biomarkers and the biological characteristics of clear cell carcinoma. Therefore, further investigation is required to clarify which of the identified biomarkers is the most interesting or important.

In conclusion, the present literature review selected a diagnostic panel of potentially useful markers for distinguishing clear cell carcinoma from high-grade serous adenocarcinoma. Additional investigation is required to determine whether these gene products are elevated in blood samples, and whether they will ultimately be useful in the diagnosis and monitoring of patients with ovarian clear cell carcinoma.

\section{Acknowledgements}

The present review was supported by a grant-in-aid for Scientific Research received from the Ministry of Education, Science and Culture of Japan, and granted to Professor Hiroshi Kobayashi of the Department of Obstetrics and Gynecology, Nara Medical University (Kashihara, Japan).

\section{References}

1. Jacobs IJ and Menon U: Progress and challenges in screening for early detection of ovarian cancer. Mol Cell Proteomics 3: 355-366, 2004

2. Nozawa S, Yajima M, Sakuma T, et al: Cancer-associated galactosyltransferase as a new tumor marker for ovarian clear cell carcinoma. Cancer Res 50: 754-759, 1990.

3. Haruta S, Furukawa N, Yoshizawa Y, et al: Molecular genetics and epidemiology of epithelial ovarian cancer (Review). Oncol Rep 26: 1347-1356, 2011.

4. Gadducci A, Lanfredini $\mathrm{N}$ and Tana R: Novel insights on the malignant transformation of endometriosis into ovarian carcinoma. Gynecol Endocrinol 30: 612-617, 2014.

5. Lalwani N, Prasad SR, Vikram R, Shanbhogue AK, Huettner PC and Fasih N: Histologic, molecular, and cytogenetic features of ovarian cancers: Implications for diagnosis and treatment. Radiographics 31: 625-646, 2011.

6. Ahmed AA, Etemadmoghadam D, Temple J, et al: Driver mutations in TP53 are ubiquitous in high grade serous carcinoma of the ovary. J Pathol 221: 49-56, 2010.

7. Uekuri C, Shigetomi H, Ono S, Sasaki Y, Matsuura M and Kobayashi H: Toward an understanding of the pathophysiology of clear cell carcinoma of the ovary (Review). Oncol Lett 6 : 1163-1173, 2013.

8. Shimizu M, Nikaido T, Toki T, Shiozawa T and Fujii S: Clear cell carcinoma has an expression pattern of cell cycle regulatory molecules that is unique among ovarian adenocarcinomas Cancer 85: 669-677, 1999.

9. Shigetomi H, Sudo T, Shimada K, et al: Inhibition of cell death and induction of G2 arrest accumulation in human ovarian clear cells by HNF-1 $\beta$ transcription factor: Chemosensitivity is regulated by checkpoint kinase CHK1. Int J Gynecol Cancer 24: $838-843,2014$

10. Shigetomi H, Higashiura Y, Kajihara $\mathrm{H}$ and Kobayashi $\mathrm{H}$ : A potential link of oxidative stress and cell cycle regulation for development of endometriosis. Gynecol Endocrinol 28: 897-902, 2012.

11. Gütgemann I, Lehman NL, Jackson PK and Longacre TA: Emil protein accumulation implicates misregulation of the anaphase promoting complex/cyclosome pathway in ovarian clear cell carcinoma. Mod Pathol 21: 445-454, 2008.
12. Min KW, Park MH, Hong SR, et al; Gynecologic Pathology Study Group of the Korean Society of Pathologists. Clear cell carcinomas of the ovary: A multi-institutional study of 129 cases in Korea with prognostic significance of Emi1 and Galectin-3. Int J Gynecol Pathol 32: 3-14, 2013.

13. Arnason T, Pino MS, Yilmaz O, et al: Cables1 is a tumor suppressor gene that regulates intestinal tumor progression in Apc(Min) mice. Cancer Biol Ther 14: 672-678, 2013.

14. Lin F, Zhang PL, Yang XJ, et al: Human kidney injury molecule-1 (hKIM-1): A useful immunohistochemical marker for diagnosing renal cell carcinoma and ovarian clear cell carcinoma. Am J Surg Pathol 31: 371-381, 2007.

15. Tsuda H, Bandera CA, Birrer MJ, Hashiguchi Y, Berkowitz RS and Mok SC: Cyclin E amplification and overexpression in clear cell adenocarcinoma of the ovary. Oncology 67: 291-299, 2004

16. Kaneuchi M, Sasaki M, Tanaka Y, et al: Expression and methylation status of 14-3-3 sigma gene can characterize the different histological features of ovarian cancer. Biochem Biophys Res Commun 316: 1156-1162, 2004.

17. Alkatout I, Friemel J, Sitek B, Anlauf M, Eisenach PA, Stühler K, Scarpa A, Perren A, Meyer HE, Knoefel WT, et al: Novel prognostic markers revealed by a proteomic approach separating benign from malignant insulinomas. Mod Pathol 28: 69-79, 2015.

18. Byrne JA, Maleki S, Hardy JR, et al: MAL2 and tumor protein D52 (TPD52) are frequently overexpressed in ovarian carcinoma, but differentially associated with histological subtype and patient outcome. BMC Cancer 10: 497, 2010.

19. Campbell IG, Russell SE, Choong DY, et al: Mutation of the PIK3CA gene in ovarian and breast cancer. Cancer Res 64: 7678-7681, 2004

20. Kuo KT, Mao TL, Jones S, et al: Frequent activating mutations of PIK3CA in ovarian clear cell carcinoma. Am J Pathol 174: 1597-1601, 2009

21. Yamamoto S, Tsuda H, Miyai K, Takano M, Tamai S and Matsubara O: Gene amplification and protein overexpression of MET are common events in ovarian clear-cell adenocarcinoma: Their roles in tumor progression and prognostication of the patient. Mod Pathol 24: 1146-55, 2011.

22. Sugita S, Morishita Y, Kano J, Furuya S, Shiba-Ishii A and Noguchi M: IGFBP-1 is expressed specifically in ovarian clear cell adenocarcinoma. Histopathology 58: 729-738, 2011.

23. Wang H, Rosen DG, Wang H, Fuller GN, Zhang W and Liu J: Insulin-like growth factor-binding protein 2 and 5 are differentially regulated in ovarian cancer of different histologic types. Mod Pathol 19: 1149-1156, 2006.

24. Bell JL, Wächter K, Mühleck B, et al: Insulin-like growth factor 2 mRNA-binding proteins (IGF2BPs): Post-transcriptional drivers of cancer progression? Cell Mol Life Sci 70: 2657-2675, 2013.

25. Boonjaraspinyo S, Boonmars T, Wu Z, et al: Platelet-derived growth factor may be a potential diagnostic and prognostic marker for cholangiocarcinoma. Tumour Biol 33: 1785-1802, 2012.

26. Yamamoto S, Tsuda H, Takano M, et al: Expression of platelet-derived growth factors and their receptors in ovarian clear-cell carcinoma and its putative precursors. Mod Pathol 21: $115-124,2008$

27. Prentice LM, Klausen C, Kalloger S, et al: Kisspeptin and GPR54 immunoreactivity in a cohort of 518 patients defines favourable prognosis and clear cell subtype in ovarian carcinoma. BMC Med 15: 5-33, 2007.

28. Canbay E, Ergen A, Bugra D, et al: Kisspeptin-54 levels are increased in patients with colorectal cancer. World J Surg 36: 2218-2224, 2012.

29. Fujimura M, Katsumata N, Tsuda H, et al: HER2 is frequently over-expressed in ovarian clear cell adenocarcinoma: Possible novel treatment modality using recombinant monoclonal antibody against HER2, trastuzumab. Jpn J Cancer Res 93: $1250-1257,2002$

30. Taniguchi F, Itamochi H, Harada T and Terakawa N: Fibroblast growth factor receptor 2 expression may be involved in transformation of ovarian endometrioma to clear cell carcinoma of the ovary. Int J Gynecol Cancer 23: 791-796, 2013.

31. Maretzky T, Le Gall SM, Worpenberg-Pietruk S, et al: Src stimulates fibroblast growth factor receptor- 2 shedding by an ADAM15 splice variant linked to breast cancer. Cancer Res 69: 4573-4576, 2009.

32. Li W, Wang H, Jin X and Zhao L: Loss of RhoGDI is a novel independent prognostic factor in hepatocellular carcinoma. Int J Clin Exp Pathol 6: 2535-2541, 2013. 
33. Canet B, Pons C, Espinosa I and Prat J: Ovarian clear cell carcinomas: RHO GTPases may contribute to explain their singular biologic behavior. Hum Pathol 42: 833-839,2011.

34. Clayton EF, Ziober A, Yao Y and Bing Z. Malignant tumors with clear cell morphology: A comparative immunohistochemical study with renal cell carcinoma antibody, Pax8, steroidogenic factor 1, and brachyury. Ann Diagn Pathol 17: 192-197, 2013.

35. Tsuchiya A, Sakamoto M, Yasuda J, et al: Expression profiling in ovarian clear cell carcinoma: Identification of hepatocyte nuclear factor-1 beta as a molecular marker and a possible molecular target for therapy of ovarian clear cell carcinoma. Am J Pathol 163: 2503-2512, 2003.

36. Senkel S, Lucas B, Klein-Hitpass L and Ryffel GU: Identification of target genes of the transcription factor HNF1beta and HNF1alpha in a human embryonic kidney cell line. Biochim Biophys Acta 1731: 179-190, 2005.

37. Hirasawa A, Saito-Ohara F, Inoue J, et al: Association of 17q21-q24 gain in ovarian clear cell adenocarcinomas with poor prognosis and identification of PPM1D and APPBP2 as likely amplification targets. Clin Cancer Res 9: 1995-2004, 2003.

38. Liang L, Li Q, Huang LY, Li W, Wang YW, Li XX and Cai SJ: Loss of ARHGDIA expression is associated with poor prognosis in HCC and promotes invasion and metastasis of HCC cells. Int J Oncol 45: 659-666, 2014.

39. Nezhat F, Cohen C, Rahaman J, Gretz H, Cole P and Kalir T: Comparative immunohistochemical studies of bcl-2 and p53 proteins in benign and malignant ovarian endometriotic cysts. Cancer 94: 2935-2940, 2002.

40. Nomura H, Tamada Y, Miyagi T, et al: Expression of NEU3 (plasma membrane-associated sialidase) in clear cell adenocarcinoma of the ovary: Its relationship with $\mathrm{T}$ factor of pTNM classification. Oncol Res 16: 289-297, 2006.

41. Sönmez H, Süer S, Güngör Z, Baloglu H and Kökoglu E: Tissue and serum sialidase levels in breast cancer. Cancer Lett 136 75-78,1999.

42. Spowart JE, Townsend KN, Huwait H, et al: The Autophagy Protein LC3A Correlates with Hypoxia and is a Prognostic Marker of Patient Survival in Clear Cell Ovarian Cancer. J Pathol 228: 437-447, 2012.

43. Cuff J, Salari K, Clarke N, Esheba GE, Forster AD, Huang S, West RB, Higgins JP, Longacre TA and Pollack JR: Integrative bioinformatics links HNF1B with clear cell carcinoma and tumor-associated thrombosis. PLoS One 8: e74562, 2013.

44. Kato M, Yamamoto S, Takano M, Matsubara O and Furuya K Aberrant expression of the mammalian target of rapamycin, hypoxia-inducible factor- $1 \alpha$, and glucose transporter 1 in the development of ovarian clear-cell adenocarcinoma. Int J Gynecol Pathol 31: 2542-63, 2012.

45. Mahata P: Biomarkers for epithelial ovarian cancers. Genome Inform 17: 184-193. 2006

46. Lin F, Shi J, Liu H, et al: Immunohistochemical detection of the von Hippel-Lindau gene product (pVHL) in human tissues and tumors: A useful marker for metastatic renal cell carcinoma and clear cell carcinoma of the ovary and uterus. Am J Clin Pathol 129: 592-605, 2008.

47. Mehla K and Singh PK: MUC1: A novel metabolic master regulator. Biochim Biophys Acta 1845: 126-135, 2014.

48. Li B, Jin H, Yu Y, et al: HOXA10 is overexpressed in human ovarian clear cell adenocarcinoma and correlates with poor survival. Int J Gynecol Cancer 19: 1347-1352, 2009

49. Miyamoto M, Takano M, Iwaya K, et al: X-chromosome-linked inhibitor of apoptosis as a key factor for chemoresistance in clear cell carcinoma of the ovary. Br J Cancer 110: 2881-2886, 2014.

50. Skirnisdottir I, Bjersand K, Akerud H and Seidal T: Napsin A as a marker of clear cell ovarian carcinoma. BMC Cancer 13 $524,2013$.

51. Jiang L, Siu MK, Wong OG, et al: iASPP and chemoresistance in ovarian cancers: Effects on paclitaxel-mediated mitotic catastrophe. Clin Cancer Res 17: 6924-6933, 2011

52. Maeda D, Ota S, Takazawa Y, et al: Glypican-3 expression in clear cell adenocarcinoma of the ovary. Mod Pathol 22: 824-832, 2009

53. Suzuki S, Yoshikawa T, Hirosawa T, et al: Glypican-3 could be an effective target for immunotherapy combined with chemotherapy against ovarian clear cell carcinoma. Cancer Sci 102 1622-1629, 2011.

54. Tsuda H, Ito YM, Ohashi Y, et al: Identification of overexpression and amplification of ABCF2 in clear cell ovarian adenocarcinomas by cDNA microarray analyses. Clin Cancer Res 11: 6880-6888, 2005 .
55. Lee HJ, Do JH, Bae S, et al: Immunohistochemical evidence for the over-expression of Glutathione peroxidase 3 in clear cell type ovarian adenocarcinoma. Med Oncol 28 (Suppl 1): S522-S527, 2011

56. Miao Y, Cai B, Liu L, Yang Y and Wan X: Annexin IV is differentially expressed in clear cell carcinoma of the ovary. Int J Gynecol Cancer 19: 1545-1549, 2009.

57. Kuroda T, Hirohashi Y, Torigoe T, et al: ALDH1-high ovarian cancer stem-like cells can be isolated from serous and clear cell adenocarcinoma cells, and ALDH1 high expression is associated with poor prognosis. PLoS One 8: e65158, 2013.

58. Kato N, Toukairin M, Asanuma I and Motoyama T: Immunocytochemistry for hepatocyte nuclear factor-1beta (HNF-1beta): A marker for ovarian clear cell carcinoma. Diagn Cytopathol 35: 193-197, 2007.

59. Arakawa N, Miyagi E, Nomura A, et al: Secretome-based identification of TFPI2, a novel serum biomarker for detection of ovarian clear cell adenocarcinoma. J Proteome Res 12: 4340-4350, 2013

60. Abu Saadeh F, Norris L, O'Toole S, et al: Tumour expresion of tissue factor and tissue factor pathway inhibitor in ovarian cancer-relationship with venous thrombosis risk. Thromb Res 132: 627-634, 2013.

61. Yamamoto S, Konishi I, Mandai M, et al: Expression of vascular endothelial growth factor (VEGF) in epithelial ovarian neoplasms: Correlation with clinicopathology and patient survival, and analysis of serum VEGF levels. Br J Cancer 76: 1221-1227, 1997.

62. Yoffou PH, Edjekouane L, Meunier L, et al: Subtype specific elevated expression of hyaluronidase-1 (HYAL-1) in epithelial ovarian cancer. PLoS One 6: e20705, 2011.

63. Quattrocchi L, Sisson M, Green A, Martin SG, Durrant L and Deen S: Expression of angiogenic chemokines in ovarian clear cell carcinoma. J Obstet Gynaecol Res 39: 297-304, 2013.

64. Suh EJ, Kabir MH, Kang UB, et al: Comparative profiling of plasma proteome from breast cancer patients reveals thrombospondin-1 and BRWD3 as serological biomarkers. Exp Mol Med 44: 36-44, 2012.

65. Sundar IK, Yao $\mathrm{H}$ and Rahman I: Oxidative stress and chromatin remodeling in chronic obstructive pulmonary disease and smoking-related diseases. Antioxid Redox Signal 18: 1956-1971, 2013

66. Samartzis EP, Noske A, Dedes KJ, Fink D and Imesch P: ARID1A mutations and PI3K/AKT pathway alterations in endometriosis and endometriosis-associated ovarian carcinomas. Int J Mol Sci 14: 18824-18849, 2013

67. Nishimura S, Tsuda H, Ito K, et al: Differential expression of hypoxia-inducible protein 2 among different histological types of epithelial ovarian cancer and in clear cell adenocarcinomas. Int J Gynecol Cancer 20: 220-226, 2010.

68. Balan V, Wang Y, Nangia-Makker P, et al: Galectin-3: A possible complementary marker to the PSA blood test. Oncotarget 4: 542-549, 2013.

69. Ho CM, Lai HC, Huang SH, Chien TY, Lin MC and Chang SF: Promoter methylation of sFRP5 in patients with ovarian clear cell adenocarcinoma. Eur J Clin Invest 40: 310-318, 2010.

70. Saeki H, Hashizume A, Izumi H, et al: The utility of serum $\mathrm{N}$-ERC/mesothelin as a biomarker of ovarian carcinoma. Oncol Lett 4: 637-641, 2012

71. Ho CM, Cheng WF, Lin MC, et al: Prognostic and predictive values of E-cadherin for patients of ovarian clear cell adenocarcinoma. Int J Gynecol Cancer 20: 1490-1497, 2010.

72. Kusumoto T, Kodama J, Seki N, Nakamura K, Hongo A and Hiramatsu Y: Clinical significance of syndecan-1 and versican expression in human epithelial ovarian cancer. Oncol Rep 23: 917-925, 2010

73. Yamaguchi K, Mandai M, Oura T, et al: Identification of an ovarian clear cell carcinoma gene signature that reflects inherent disease biology and the carcinogenic processes. Oncogene 29: $1741-1752,2010$

74. Kato N, Sasou S, Teshima S and Motoyama T: Overexpression of laminin-5 gamma2 chain in clear cell carcinoma of the ovary. Virchows Arch 450: 273-278, 2007.

75. Akahane T, Sekizawa A, Okuda T, Kushima M, Saito H and Okai T: Disappearance of steroid hormone dependency during malignant transformation of ovarian clear cell cancer. Int J Gynecol Pathol 24: 369-376, 2005.

76. Oe S, Hasegawa K, Nagase S, Kato R, Torii Y and Udagawa Y: Expression of podoplanin in epithelial ovarian carcinomas and its potential as a marker for clear cell adenocarcinoma. Int $\mathbf{J}$ Gynecol Pathol 29: 405-410, 2010. 